\title{
Óleo de aroeira-vermelha sobre o desempenho e a morfometria intestinal de frangos de corte
}

\author{
Brazilian red pepper oil on the performance and intestinal morphometry of broilers
}

\author{
Maria Aparecida da Silva ${ }^{\mathrm{I}}$ Bruna Mirelly de Sousa Pessotti ${ }^{\mathrm{II}}$ Surama Freitas Zanini ${ }^{\mathrm{II}}$ \\ Geraldo Luiz Colnago $^{\mathrm{IV}}$ Louisiane de Carvalho Nunes ${ }^{\mathrm{III}}$ Maria Regina Alves Rodrigues ${ }^{\mathrm{V}}$ \\ Larissa Ferreira ${ }^{\mathrm{VI}}$
}

\section{RESUMO}

\begin{abstract}
Avaliou-se a utilização do óleo essencial extraído dos frutos de aroeira-vermelha (Schinus terebinthifolius Raddi) na dieta de frangos de corte sobre o desempenho e a morfometria intestinal. Utilizaram-se 240 pintos de um dia de idade, machos, da linhagem Cobb, distribuídos em delineamento inteiramente casualizado em grupos de cinco tratamentos e quatro repetições: sem antibiótico $e$ anticoccidiano (controle negativo); com antibiótico $e$ anticoccidiano (controle positivo); com $0,1 \%$ de óleo de aroeira; com 0,2\% de óleo de aroeira; e com 0,4\% de óleo de aroeira. $O$ consumo de ração, peso corporal e conversão alimentar foram influenciados significativamente pelos tratamentos $(P<0,05)$. As aves tratadas com promotor de crescimento (antibiótico) tiveram desempenho superior ao desempenho das aves não tratadas $(P<0,05)$. De 1-21 dias de idade, a adição de 0,4\% de óleo de aroeira resultou em melhoria na conversão alimentar e maior ganho de peso $(P<0,05)$. De 22-47 dias de idade, observou-se que, independente do nível de adição de óleo de aroeira, sua utilização resultou em maior ganho de peso e peso final $(P<0,05)$ e maior altura de vilosidades no jejuno das aves alimentadas com óleo de aroeira comparadas com as aves tratadas com controle negativo $(P<0,05)$. O óleo de aroeira melhorou os índices de produção e aumentou a superfície intestinal absortiva.
\end{abstract}

Palavras-chave: frangos de corte, Schinus terebinthifolius Raddi, óleo de aroeira-vermelha, desempenho, morfometria jejunal.

\begin{abstract}
The aim of this research was to evaluate the use of essential oil obtained from the fruits of Brazilian red pepper in the performance and intestinal morphometry of broilers. A total of 240 day-old male chicks Cobb were used, distributed in a randomized design in groups of five treatments and four replicates of 12 birds each: diet without antibiotics and anticoccidial agent (negative control); diet with antibiotic and anticoccidial agent (positive control); diets with $0.1 \%$ of Brazilian red pepper oil; diets with $0.2 \%$ of Brazilian red pepper oil; diets with $0.4 \%$ of Brazilian red pepper oil. Feed intake, body weight and feed conversion were influenced by treatments $(P<0.05)$. It was observed that the broilers fed with the growth promoter antibiotic had a superior performance than the untreated broilers $(P<0.05)$. In the period of $1-21$ days, the inclusion of $0.4 \%$ Brazilian red pepper oil resulted in improvements in feed conversion and increased weight gain compared to other treatments $(P<0.05)$. From 22-47 days of age it was observed that regardless of the level of Brazilian red pepper oil, their use resulted in a higher weight gain and final weight $(P<0.05)$ and it was recorded a maximum villous height in broilers fed with Brazilian red pepper oil as compared to birds not treated with antibiotics $(P<0.05)$. The Brazilian red pepper improved production rates and increased the intestinal absorptive surface.
\end{abstract}

Key words: broilers, Schinus terebinthifolius Raddi, essential oil, intestinal morphology and performance.

'Programa de Pós-graduação em Ciências Veterinárias, Universidade Federal do Espírito Santo (UFES), Alegre, ES, Brasil.

"Programa de Pós-graduação em Biotecnologia, UFES, Vitória, ES, Brasil.

"IIDepartamento de Medicina Veterinária, UFES. Alto Universitário s/n, CP 16, 29500-000, Alegre, ES, Brasil. E-mail: surama@cca.ufes.br. *Autor para correspondência.

${ }^{\mathrm{IV}}$ Departamento de Zootecnia, Universidade Federal Fluminense (UFF), Niterói, RJ, Brasil.

`Departamento de Química Orgânica, Universidade Federal de Pelotas (UFPel), Pelotas, RS, Brasil.

${ }^{\mathrm{VI} C}$ Curso de Medicina Veterinária, UFES, Vitória, ES, Brasil. 


\section{INTRODUÇÃO}

Tem sido verificado um crescente interesse em avaliar os extratos de plantas, principalmente os óleos essenciais, no controle das infecções entéricas. Entre os possíveis mecanismos de ação dos óleos essenciais no organismo animal, destacam-se a modulação na microbiota intestinal, a melhoria da resposta imune, o controle na produção de amônia, o aumento na digestibilidade e a absorção de nutrientes, a partir do aumento da produção de enzimas e secreções intestinais, além da manutenção da integridade do epitélio intestinal (BRUGALLI, 2003).

Sabe-se que, quando um patógeno causa lesão nas células epiteliais intestinais, pode haver modificações na capacidade de digestão e absorção de nutrientes com o surgimento de inflamações crônicas na mucosa intestinal (OLIVEIRA, 1998), que pode resultar em diminuição das vilosidades, aumento do turnover celular e redução da atividade digestiva e absortiva (BOLELI et al., 2002).

A aroeira-vermelha (Schinus terebinthifolius Raddi) é uma espécie vegetal pertencente à família Anacardiaceae R. Br. Seu óleo essencial não mostrou nenhuma toxicidade em animais e humanos (BARBOSA et al., 2007). As pesquisas realizadas com a Schinus terebenthifolius Raddi se concentram na análise das folhas e da seiva (tronco), e poucas pesquisas foram encontradas utilizando os frutos da aroeira.

De acordo com BARBOSA et al. (2007), o óleo essencial de aroeira-vermelha é constitituído principalmente de monoterpenos $(90,00 \%)$, com uma maior concentração de $\delta$-3-careno $(29,22 \%)$, $\alpha$-pineno $(12,94 \%), \alpha$-felandreno $(13,04 \%)$ e $\beta$-felandreno $(18,08 \%)$, embora também tenha sido observada a ocorrência de sesquiterpenos, como o D-germacreno $(3,09 \%)$. Anteriormente, SINGH et al. (1998) relataram que a maioria das amostras analisadas de óleo de aroeira-vermelha revelaram o a-pineno $(15,01-51,82 \%)$ como o principal componente além do $\alpha$-felandreno, elixeno, D-germacreno, limoneno e $p$-cimeno.

Considerando as possíveis implicações dessa ação antimicrobiana, objetivou-se avaliar níveis crescentes de óleo essencial extraído de frutos de aroeira-vermelha (Schinus terebinthifolius Raddi) na dieta sobre o desempenho e a integridade da mucosa intestinal absortiva de frangos de corte.

\section{MATERIAL E MÉTODOS}

O óleo essencial de frutos de aroeiravermelha (Schinus terebinthifolius Raddi) foi obtido pela técnica de destilação por arraste a vapor de acordo com BERTOLDI (2006). As amostras do óleo essencial de aroeira-vermelha foram submetidas à análise por Cromatografia Gasosa acoplada à Espectrometria de Massa (CG-MS), em um equipamento GC-MS (Schimadzu, modelo 5050A) equipado com uma coluna de sílica OV-05 (30mx0,25mmx0,25 $\mu \mathrm{m})$, nas seguintes condições cromatográficas: a temperatura inicial foi de $60^{\circ} \mathrm{C}$, aumentando a temperatura em $4^{\circ} \mathrm{C} \mathrm{min}^{-1}$, até atingir $280^{\circ} \mathrm{C}$, permanecendo nessa temperatura por $10 \mathrm{~min}$; Temperatura do detector $=280^{\circ} \mathrm{C} ; \mathrm{T}$ Temperatura do injetor $=280^{\circ} \mathrm{C} ; \mathrm{T}$ Temperatura da coluna $=60^{\circ} \mathrm{C}$; Split $=1: 50 ; \mathrm{E}=2,0 \mathrm{kV}$; fluxo $=1,0 \mathrm{~mL} \mathrm{~m}^{-1} ; \mathrm{e}$ modo $=\mathrm{SCAN}$. Foi injetado $1 \mu \mathrm{L}$ das soluções do óleo essencial (1000mg L $\mathrm{m}^{-1}$ em hexano) e dos 15 padrões cromatográficos $\alpha$-pineno, canfeno, $\beta$-pineno, mirceno, $\alpha$-terpineno, $p$-cimeno, limoneno, 1,8-cineol, (terpineno, terpinoleno, linalol, 4-terpineol, $\alpha$-terpineol, timol e carvacrol (40mg L $\mathrm{m}^{-1}$ em hexano), e os constituintes foram identificados por comparação com os espectros de massa da biblioteca Wiley do equipamento e o tempo de retenção dos padrões. A concentração dos monoterpenos encontrados nas amostras de óleo essencial dos frutos de aroeiravermelha foi calculada em relação à área normalizada dos picos, cujos tempos de retenção foram iguais aos tempos dos padrões. Essa concentração não foi expressa em massa, mas como porcentagem em área.

Foram utilizados 240 pintos de corte de um dia, machos, da linhagem Cobb, alojados em 20 boxes de $2 \mathrm{~m}^{2}$ (12 aves boxe $\left.{ }^{-1}\right)$, distribuídos em um delineamento inteiramente casualizado, em grupos de cinco tratamentos e quatro repetições, com 12 aves cada: T1: dieta com antibiótico e anticoccidiano (controle positivo); T2: dieta sem antibiótico e anticoccidiano (controle negativo); T3: dieta com 0,1\% de óleo de aroeira-vermelha; T4: dieta com $0,2 \%$ de óleo de aroeira-vermelha; e T5: dieta com $0,4 \%$ de óleo de aroeira-vermelha. No período de um a 21 dias de idade, os animais foram criados com uma ração inicial com $22 \%$ de PB e $2900 \mathrm{kcal} \mathrm{EM} \mathrm{kg}^{-1}$. Do 22ㅇ ao 47ㅇ dia de idade, as aves foram alimentadas com a ração de crescimento/terminação com 19\% PB e 3000kcal EM $\mathrm{kg}^{-1}$ à base de milho e soja. A bacitracina de zinco $15 \%$ $\left(45 \mathrm{mg} \mathrm{kg}^{-1}\right)$ e salinomicina $12 \%\left(66 \mathrm{mg} \mathrm{kg}^{-1}\right)$ foram o antibiótico e o anticoccidiano, respectivamente, utilizados na dieta do grupo controle positivo.

As aves foram pesadas no início e no final de cada período experimental (aos 21 e 47 dias de idade), visando à determinação do ganho de peso. No cálculo do consumo de ração, consideraram-se a ração fornecida, os desperdícios e as sobras nos comedouros, ocorridos durante o período experimental. Assim, o 
consumo de ração foi obtido com base no consumo médio por unidade experimental dentro de cada fase. Os dados de conversão alimentar foram obtidos por meio da divisão do consumo médio de ração pelo ganho médio de peso, por fase.

No $14^{\circ}$ e $21^{\circ}$ dias de idade, quatro aves por tratamento foram retiradas de acordo com o peso médio do boxe e abatidas por meio de deslocamento cervical para coleta de fragmentos de jejuno com quatro centímetros de comprimento. Esse material foi imediatamente lavado em solução fisiológica e fixado em solução de Bouin. Os fragmentos foram submetidos ao processo de inclusão em parafina de acordo com a técnica histopatológica de rotina. Secções com espessura de $7 \mu \mathrm{m}$ foram coradas com hematoxilinaeosina. Foram feitas mensurações no jejuno, de acordo com MAIORKA et al. (2000), da altura da vilosidade intestinal, desde o epitélio luminal até a camada muscular da mucosa e da profundidade da cripta, medida do epitélio luminal da cripta até a muscular da mucosa e a relação vilo:cripta. A morfometria do jejuno foi realizada utilizando-se um microscópio óptico com aumento de 100 vezes (objetiva 10x). A captura de imagem foi feita com Câmera Olympus Polaroid e sua análise foi feita a partir do software Sigma Scan Pro 5.0.

As análises estatísticas dos parâmetros avaliados foram realizadas com utilização do programa SAEG (Sistema para Análises Estatísticas e Genéticas) (UFV, 1997). Os resultados foram submetidos à análise de variância utilizando o teste de Tukey para comparação de médias entre os tratamentos $(\mathrm{P}<0,05)$.

\section{RESULTADOS E DISCUSSÃO}

Os resultados deste estudo revelaram que, entre os compostos presentes no óleo essencial de aroeira-vermelha, obtidos de frutos maduros, o $\alpha$ pineno foi o constituinte majoritário $(29,39 \%)$, seguido pelo $\Delta$-careno $(19,69 \%)$, limoneno $(18,15 \%)$ e $\alpha$ felandreno (9,39\%), corroborando SINGH et al. (1998) e BARBOSA et al. (2007). Assim, verificou-se uma grande concentração de monoterpenos e ausência de sesquiterpenos (Tabela 1). Os óleos essenciais são obtidos por métodos de destilação, usualmente por arraste a vapor, e são constituídos por uma mistura de terpenoides, monoterpenos e sesquiterpenos, taninos e uma variedade de hidrocarbonetos alifáticos de baixo peso molecular, ácidos, alcoóis, aldeídos, cumarinas e éster acíclico (DORMAN \& DEANS, 2000).

Na tabela 2 encontram-se os dados referentes ao desempenho no período de 1-21 e 22-47 dias de idade. Durante 1-21 dias de idade, o consumo de ração não foi influenciado pelos tratamentos ( $\mathrm{P}>0,05)$, mas a conversão alimentar, o ganho de peso e o peso final dos frangos tratados com óleo de aroeiravermelha foram superiores ao desempenho apresentado pelos frangos tratados sem promotor de crescimento $(\mathrm{P}<0,05)$. A maximização nesses índices zootécnicos parece indicar uma melhoria nos processos de digestão e absorção de nutrientes, tornando-os mais eficientes, como foi visualizado pela maior altura de vilosidades de jejuno das aves arraçoadas com óleo de aroeiravermelha em comparação com as aves tratadas sem promotor de crescimento $(\mathrm{P}<0,05$; Tabela 3$)$.

Tabela 1 - Relação dos padrões de terpênicos e dos compostos encontrados na amostra de óleo essencial de aroeira-vermelha (Schinus terebinthifolius Raddi) submetidos à cromatografia gasosa com detector espectrométrico de massa.

\begin{tabular}{lccclc}
\hline Pico & Tempo retenção $(\mathrm{min})$ & Fórmula molecular & Peso molecular & Composto & Concentração** (\%) \\
\hline 1 & 5,64 & $\mathrm{C}_{10} \mathrm{H}_{16}$ & 136 & $\alpha$-tujeno & 0,67 \\
2 & 5,85 & $\mathrm{C}_{10} \mathrm{H}_{16}$ & 136 & $\alpha$-pineno* & 29,39 \\
3 & $\mathrm{C}_{10} \mathrm{H}_{16}$ & 136 & Canfeno* & 0,31 \\
4 & 5,69 & $\mathrm{C}_{10} \mathrm{H}_{16}$ & 136 & Sabineno & 3,99 \\
5 & 6,94 & $\mathrm{C}_{10} \mathrm{H}_{16}$ & 136 & $\beta$-pineno* & 7,71 \\
6 & 7,06 & $\mathrm{C}_{10} \mathrm{H}_{16}$ & 136 & Mirceno* & 6,18 \\
7 & 7,43 & $\mathrm{C}_{10} \mathrm{H}_{16}$ & 136 & $\alpha$-felandreno & 9,39 \\
8 & 7,90 & $\mathrm{C}_{10} \mathrm{H}_{16}$ & 136 & $\delta$-Careno & 19,69 \\
9 & 8,12 & $\mathrm{C}_{10} \mathrm{H}_{16}$ & 136 & $\alpha$-terpineno* & 0,25 \\
10 & 8,30 & $\mathrm{C}_{10} \mathrm{H}_{14}$ & 134 & -cimeno* & 4,27 \\
11 & 8,56 & $\mathrm{C}_{10} \mathrm{H}_{16}$ & 136 & Limoneno* & 18,15 \\
\hline
\end{tabular}

* compostos identificados com base nos padrões cromatográficos.

** concentração calculada em relação à área normalizada dos picos. 
Tabela 2 - Desempenho de frangos de corte alimentados com dieta contendo diferentes níveis de óleo de aroeira durante os períodos de 1-21 e 22-47 dias.

\begin{tabular}{|c|c|c|}
\hline Dietas & $1-21$ dias & $22-47$ dias \\
\hline & \multicolumn{2}{|c|}{ Consumo de ração $\left(\mathrm{g}_{\text {ave }}{ }^{-1}\right)$} \\
\hline Controle negativo (sem antibiótico + anticoccidiano) & $927,28 \pm 37,28$ & $1662,2 \pm 243,8 \mathrm{~B}$ \\
\hline Controle positivo (antibiótico + anticoccidiano) & $909,49 \pm 52,83$ & $3157,2 \pm 132,4 \mathrm{~A}$ \\
\hline $0,1 \%$ de óleo de aroeira & $944,83 \pm 22,59$ & $3311,5 \pm 251,6 \mathrm{~A}$ \\
\hline $0,2 \%$ de óleo de aroeira & $941,93 \pm 82,46$ & $3173,2 \pm 178,0 \mathrm{~A}$ \\
\hline $0,4 \%$ de óleo de aroeira & $909,53 \pm 61,28$ & $3225,2 \pm 209,5 \mathrm{~A}$ \\
\hline \multirow[t]{2}{*}{$\mathrm{CV}(\%)$} & 5,9 & 3,2 \\
\hline & \multicolumn{2}{|c|}{ Ganho de peso $\left(\mathrm{g} \mathrm{ave}^{-1}\right)$} \\
\hline Controle negativo (sem antibiótico + anticoccidiano) & $450,1 \pm 19,3 \mathrm{~B}$ & $333,8 \pm 51,3 \mathrm{C}$ \\
\hline Controle positivo (antibiótico + anticoccidiano) & $482,6 \pm 18,3 \mathrm{AB}$ & $1669,5 \pm 114,3 \mathrm{~B}$ \\
\hline $0,1 \%$ de óleo de aroeira & $515,68 \pm 23,7 \mathrm{~A}$ & $1772,8 \pm 145,7 \mathrm{~A}$ \\
\hline $0,2 \%$ de óleo de aroeira & $477,10 \pm 39,5 \mathrm{AB}$ & $1764,5 \pm 146,2 \mathrm{~A}$ \\
\hline $0,4 \%$ de óleo de aroeira & $519,93 \pm 30,8 \mathrm{~A}$ & $1778,1 \pm 108,1 \mathrm{~A}$ \\
\hline \multirow[t]{2}{*}{$\mathrm{CV}(\%)$} & 5,6 & 3,07 \\
\hline & \multicolumn{2}{|c|}{ Peso vivo (g/ave) } \\
\hline Controle negativo (sem antibiótico + anticoccidiano) & $523,35 \pm 18,8 \mathrm{~B}$ & $857,2 \pm 62,5 \mathrm{C}$ \\
\hline Controle positivo (antibiótico + anticoccidiano) & $555,4 \pm 18,5 \mathrm{AB}$ & $2225,2 \pm 100,7 \mathrm{~B}$ \\
\hline $0,1 \%$ de óleo de aroeira & $588,33 \pm 24,2 \mathrm{~A}$ & $2361,2 \pm 141,7 \mathrm{~A}$ \\
\hline $0,2 \%$ de óleo de aroeira & $550,3 \pm 39,2 \mathrm{AB}$ & $2315,0 \pm 171 \mathrm{AB}$ \\
\hline $0,4 \%$ de óleo de aroeira & $593,12 \pm 30,5 \mathrm{~A}$ & $2371,3 \pm 117,2 \mathrm{~A}$ \\
\hline \multirow[t]{2}{*}{$\mathrm{CV}(\%)$} & 4,8 & 3,08 \\
\hline & \multicolumn{2}{|c|}{ Conversão alimentar } \\
\hline Controle negativo (sem antibiótico + anticoccidiano) & $2,07 \pm 0,03 \mathrm{~A}$ & $5,44 \pm 0,7 \mathrm{~A}$ \\
\hline Controle positivo (antibiótico + anticoccidiano) & $1,93 \pm 0,04 \mathrm{~B}$ & $1,91 \pm 0,08 \mathrm{~B}$ \\
\hline $0,1 \%$ de óleo de aroeira & $1,86 \pm 0,11 \mathrm{BC}$ & $1,90 \pm 0,05 \mathrm{~B}$ \\
\hline $0,2 \%$ de óleo de aroeira & $1,93 \pm 0,04 \mathrm{~B}$ & $1,84 \pm 0,10 \mathrm{~B}$ \\
\hline $0,4 \%$ de óleo de aroeira & $1,76 \pm 0,05 \mathrm{C}$ & $1,81 \pm 0,11 \mathrm{~B}$ \\
\hline $\mathrm{CV}(\%)$ & 3,4 & 9,66 \\
\hline
\end{tabular}

${ }^{A, B, C}$ Médias seguidas por letras distintas na mesma coluna diferem pelo teste Tukey $(\mathrm{P}<0,05)$.

A mucosa intestinal deve apresentar características morfofuncionais adequadas, pois os processos de absorção são dependentes da integridade do epitélio. Inúmeros agentes infecciosos ou não infecciosos podem lesar a mucosa intestinal, além de comprometer os processos digestórios. Atividades antiinflamatória (GAZZANEO et al., 2005) e antimicrobiana da Schinus terebinthifolius Raddi (MARTINEZ et al., 1996; MARTINEZ GUERRA et al., 2000; MELO-JUNIOR et al., 2000; SOKMEN et al., 2004; SCHMOURLO et al., 2005; LIMA et al., 2006), provavelmente atribuída à presença de substâncias fenólicas, contra uma série de microorganismos. De acordo com GOMIS et al. (2001), a proteção à saúde derivada do consumo de vegetais é atribuída, em grande parte, às propriedades biológicas do conteúdo fenólico, que incluem as atividades antioxidante, anti-inflamatória, antihistamínica, antiviral, antimicrobiana, antitumoral, anticarcinogênica e antimutagênica.
Não houve diferença significativa entre os tratamentos $(\mathrm{P}>0,05)$ para a espessura da túnica muscular, profundidade das criptas de Lieberkühn e relação vilo:cripta. De acordo com MACARI et al. (2002), a capacidade absortiva do intestino é proporcional ao número de vilosidades ali presentes. A manutenção do tamanho dos vilos garante a manutenção da capacidade digestiva e de absorção intestinal (PELICANO et al., 2003).

O frango de corte está sujeito a diversos fatores capazes de alterar as características morfofuncionais da mucosa gastrintestinal, como as lesões microbianas, que podem interferir no turnover celular e consequentemente no processo absortivo de nutrientes (MACARI et al., 2002). De acordo com LANGHOUT (2005), os óleos essenciais melhoram o desempenho dos animais por meio do aumento da palatabilidade da ração, do estímulo à secreção de enzimas endógenas, da modulação da microbiota intestinal e da redução de infecções subclínicas. 
Tabela 3 - Valores observados e expressos em micrômetros $(\mu \mathrm{m})$ da morfometria da túnica muscular, das vilosidades e das criptas de Lieberkühn do jejuno de frangos de corte alimentados com dieta contendo diferentes níveis de óleo de aroeira.

\begin{tabular}{lcc}
\hline Dietas & $14 \%$ dia de idade & $21^{\circ}$ dia de idade \\
\hline & \multicolumn{1}{c}{ Altura das vilosidades $(\mu \mathrm{m})$} \\
Controle negativo (sem antibiótico e anticoccidiano) & $1302,43 \pm 168,6$ & $1185,6 \pm 260,9 \mathrm{~B}$ \\
Controle positivo (com antibiótico e anticoccidiano) & $1497,73 \pm 236,8$ & $1819,3 \pm 421 \mathrm{AB}$ \\
$0,1 \%$ óleo de aroeira-vermelha & $1087,58 \pm 108,4$ & $1774,3 \pm 300 \mathrm{AB}$ \\
$0,2 \%$ óleo de aroeira-vermelha & $1171,14 \pm 191,3$ & $1777,6 \pm 436 \mathrm{AB}$ \\
$0,4 \%$ óleo de aroeira-vermelha & $1169,01 \pm 222,1$ & $1966,0 \pm 212,2 \mathrm{~A}$ \\
CV (\%) & 20,06 & 363 \\
& Profundidade das criptas de Lieberkühn $(\mu \mathrm{m})$ \\
Controle negativo (sem antibiótico e anticoccidiano) & $326,30 \pm 123,2$ & $366,6 \pm 101,07$ \\
Controle positivo (com antibiótico e anticoccidiano) & $326,92 \pm 10,4$ & $186,98 \pm 63,75$ \\
$0,1 \%$ óleo de aroeira-vermelha & $306,45 \pm 31,1$ & $280,87 \pm 54,03$ \\
$0,2 \%$ óleo de aroeira-vermelha & $305,72 \pm 75,8$ & $287,37 \pm 173,2$ \\
$0,4 \%$ óleo de aroeira-vermelha & $254,85 \pm 68,6$ & $242,04 \pm 16,35$ \\
CV(\%) & 24,04 & 35,72 \\
& & Relação vilo:cripta $(\mu \mathrm{m})$ \\
Controle negativo (sem antibiótico e anticoccidiano) & $4,59 \pm 0,72$ & $5,18 \pm 1,5$ \\
Controle positivo (com antibiótico e anticoccidiano) & $4,29 \pm 1,20$ & $6,55 \pm 0,9$ \\
$0,1 \%$ óleo de aroeira-vermelha & $3,55 \pm 0,02$ & $6,41 \pm 1,25$ \\
$0,2 \%$ óleo de aroeira-vermelha & $3,90 \pm 0,42$ & $7,35 \pm 3,03$ \\
$0,4 \%$ óleo de aroeira-vermelha & $4,53 \pm 0,51$ & $8,12 \pm 0,76$ \\
CV(\%) & 16,69 & 25,31 \\
\hline
\end{tabular}

${ }^{\mathrm{A}, \mathrm{B}}$ Médias seguidas por letras distintas na mesma coluna diferem pelo teste Tukey $(\mathrm{P}<0,05)$.

No período de 22-47 dias de idade, foi observado que, independente do nível de adição de óleo de aroeira-vermelha, sua utilização resultou em um maior ganho de peso e peso final em comparação com os demais tratamentos $(\mathrm{P}<0,05$; Tabela 2). Em ambos os períodos, verificou-se que os animais tratados com promotor de crescimento tiveram um desempenho superior aos não tratados $(\mathrm{P}<0,05$; Tabela 2), demonstrando a ação dos antibióticos na melhoria da taxa de crescimento e na conversão alimentar. De acordo com BRUGALLI (2003), a utilização de antibióticos na avicultura tem apresentado eficácia, especialmente quando em condições de campo de maior desafio. Vários experimentos têm relatado os efeitos benéficos dos antibióticos, indicando que os promotores de crescimento proporcionam diminuição do número de bactérias aderidas à mucosa intestinal, $\mathrm{o}$ que reduz a competição por nutrientes com o hospedeiro, diminuição de bactérias produtoras de toxinas e amônia, que prejudicam a absorção dos nutrientes, e conseqüentemente diminuição das células inflamatórias na parede intestinal e do grau de descamação e renovação das vilosidades intestinais (IZAT et al., 1989).
Sendo assim, a proliferação dos organismos patogênicos leva a um espessamento da parede intestinal e à redução do tamanho das vilosidades, uma forma de defesa, com consequente redução da eficiência absortiva intestinal, que, na prática, resulta em piora na conversão alimentar e no ganho de peso dos animais (FURLAN et al., 2004).

\section{CONCLUSÃO}

O óleo de aroeira demonstra ser uma alternativa aos quimioterápicos pela melhoria nos índices de produção e na superfície absortiva intestinal.

\section{AGRADECIMENTOS}

Ao Conselho Nacional de Desenvolvimento Científico e Tecnológico $(\mathrm{CNPq})$, pelo apoio financeiro concedido para execução desta pesquisa.

\section{COMITÊ DE ÉTICA E BIOSSEGURANÇA}

O protocolo de experimentação animal está de acordo com CONEA e foi aprovado pelo comitê de ética no uso de animais da Universidade Federal do Espírito Santo pelo protocolo $\mathrm{N}^{\circ} 01 / 08$.

Ciência Rural, v.40, n.10, out, 2010. 


\section{REFERÊNCIAS}

BARBOSA, L.C.A. et al. Seasonal variation in the composition of volatile oils from Schinus terebinthifolius Raddi. Química Nova, v.30, n.8, p.1959-1965, 2007. Disponível em: <http:// w w w. s c i e 1 o.br/s c i e 1 o.ph p ? p i d = S $0100-$ 40422007000800030\&script=sci_arttext $>$. Acesso em: 12 ago. 2010. doi: $10.1590 /$ S0100-40422007000800030.

BERTOLDI, M.C. Atividade antioxidante in vitro da fração fenólica, das oleorresinas e do óleo essencial de pimenta rosa (Schinus terebinthifolius Raddi). 2006. 96f. Dissertação (Mestrado em Ciência e Tecnologia de Alimentos) - Curso de Pós-graduação em Ciência e Tecnologia de Alimentos, Universidade Federal de Viçosa, MG.

BOLELI, I.C. et al. Estrutura funcional do trato digestório. In: MACARI, M. et al. Fisiologia aviária aplicada a frangos de corte. Jaboticabal: Funep, 2002. p.75-96.

BRUGALLI, I. Alimentação alternativa: a utilização de fitoterápicos ou nutracêuticos como moduladores da imunidade e desempenho animal. In: SIMPÓSIO SOBRE MANEJO E NUTRIÇÃO DE AVES E SUÍNOS, 2003, Campinas, SP. Anais... Campinas: CBNA, 2003. p.167-182.

DORMAN, H.J.D.; DEANS, S.G. Antimicrobial agents from plants: antibacterial activity of plant volatile oils. Journal of Applied Microbiology, v.88, n.2, p.308-316, 2000. Disponível em: <http://onlinelibrary.wiley.com/doi/10.1046/ j.1365-2672.2000.00969.x/pdf >. Acesso em: 12 ago. 2010. doi: $10.1046 / \mathrm{j} .1365-2672.2000 .00969$.

FURLAN, R.L. et al. Como avaliar os efeitos do uso de prebióticos, probióticos e flora de exclusão competitiva. In: SIMPÓSIO TÉCNICO DE INCUBAÇÃO, MATRIZES DE CORTE E NUTRIÇÃO, 5., 2004, Balneário Camboriú, SC. Anais... Balneário Camboriú: Fundação Apinco de Ciência e Tecnologias Avícolas, 2004. p. 06-28.

GAZZANEO, L.R.S. et al. Knowledge and use of medicinal plants by local specialists in a region of Atlantic Forest in the state of Pernambuco (Northeastern Brazil). Journal Ethnobiol Ethnomedicine, v.1, p.9, 2005. Disponível em: <http://www.ncbi.nlm.nih.gov/pmc/articles/PMC1291389/>. Acesso em: 12 ago. 2010. doi: 10.1186/1746-4269-1-9.

GOMIS, D.B. et al. Capillary liquid chromatographic determination of neutral phenolic compounds in apple juices. Analytica Chimica Acta, v.426, n.1, p.111-117, 2001.

IZAT, A.L. et al. Effects of dietary antibiotic treatment on yield of commercial broilers. Poultry Science, v.68, p.651$655,1989$.

LANGHOUT, P. Alternativas ao uso de quimioterápicos na dieta de aves: a visão da indústria e recentes avanços. In: CONFERENCIA APINCO DE CIÊNCIA E TECNOLOGIA AVÍCOLAS, 2005, Santos, SP. Anais... Santos: Apinco, 2005. p.21-33.
LIMA, M.R.F. et al. The antibiotic activity of some Brazilian medicinal plants. Revista Brasileira Farmacognosia, v.16, p.300-306, 2006. Disponível em: <http://www.scielo.br/ scielo.php?pid=S $0102-695 \times 2006000300004 \&$ script $=$ sci_arttext $>$. Acesso em: 12 ago. 2010. doi: 10.1590/S0102$695 \times 2006000300004$

MACARI, M. et al. Fisiologia aplicada a frangos de corte: imunologia aplicada. Jaboticabal: FUNEP/UNESP, 2002. p. 231-245.

MAIORKA, A. et al. Influência da suplementação de glutamina sobre o desempenho e o desenvolvimento de vilos e criptas do intestino delgado de frangos. Arquivo Brasileiro de Medicina Veterinária e Zootecnia, v.52, n.5, p.487-490, 2000. Disponível em: <http:// www. scielo.br/scielo.php? script=sci_arttext\&pid=S010209352000000500014>. Acesso em: 12 ago. 2010. doi: 10.1590/ S0102-09352000000500014

MARTINEZ, M.J. et al. Screening of some Cuban medicinal plants for antimicrobial activity. Journal Ethnopharmacol, v.52, p.171-174, 1996.

MARTÍNEZ GUERRA, M.J. et al. Actividad antimicrobiana de un extracto fluido al $80 \%$ de Schinus terebinthifolius Raddi (copal). Revista Cubana de Plantas Medicinales, v.5. n.1, p.23-25, 2000. Disponível em: <http://scielo.sld.cu/pdf/pla/ v5n1/pla06100.pdf >. Acesso em: 12 ago. 2010.

MELO JÚNIOR, E.J.M. et al. Estudo de plantas medicinais com atividade antimicrobiana sobre microrganismos presentes na alveolite. Revista ABO Nacional, v.8, n.4, p.220-226, 2000 .

OLIVEIRA, P.B. Influência de fatores antinutricionais de alguns alimentos sobre o epitélio intestinal e o desempenho de frangos de corte. 1998. 42f. Dissertação (Mestrado em Zootecnia) - Curso de Pós-graduação em Zootecnia, Universidade Estadual de Maringá, PR.

PELICANO, E.R.L. et al. Morfometria e ultra-estrutura da mucosa intestinal de frangos de corte alimentados com dietas contendo diferentes probióticos. Revista Portuguesa de Ciências Veterinárias, v.98, n.547, p.125-134, 2003. Disponível em: <http://www.fmv.utl.pt/spcv/PDF/pdf9_2003/ 547_125_134.pdf>. Acesso em: 12 ago. 2010.

SCHMOURLO, G. et al. Screening of antifungal agents using ethanol precipitation and bioautography of medicinal and food plants. Journal Ethnopharmacol, v.96, n.3, p.563-568, 2005.

SINGH, A.K. et al. Essential oil of leaves and inflorescence of Schinus terebinthifolius: an exotic plant of India. Journal Essential Oil Research, v.10, p.697-699, 1998.

SOKMEN, A. et al. The in vitro antimicrobial and antioxidant activities of the essential oils and methanol extracts of endemic Thymus spathulifolius. Food Control, v.15, n.8, p.627-634, 2004.

UFV - UNIVERSIDADE FEDERAL DE VIÇOSA. Manual de utilização do Programa SAEG. Viçosa, 1997. 150p. 